\title{
Travel-associated Legionnaires' disease in Europe in 2009
}

C A Joseph (cajoseph75@hotmail.com) ${ }^{1}$, K D Ricketts ${ }^{1}$, R Yadav ${ }^{1}$, S Patel ${ }^{1}$, on behalf of the European Working Group for Legionella Infections

1. Centre for Infections, Health Protection Agency, London, United Kingdom

Citation style for this article:

Joseph CA, Ricketts KD, Yadav R, Patel S, on behalf of the European Working Group for Legionella Infections. Travel-associated Legionnaires' disease in Europe in 2009. Euro Surveill. 2010;15(41):pii=19683. Available online: http://www.eurosurveillance.org/ViewArticle.aspx?Articleld=19683

Article published on 14 October 2010

A total of 818 cases of Legionnaires' disease with onset of illness in 2009 were reported from 22 European and two non-European countries to the European Surveillance Scheme for Travel-Associated Legionnaires' Disease (EWGLINET). This is a decrease of 52 cases compared with 2008 and 129 fewer than in 2007 - the peak year of reporting to date. A total of 794 (97.1\%) cases were reported as confirmed and 24 as presumptive cases. Outcome of illness was reported for $561(68.6 \%)$ cases. Of these cases 28 (5\%) were reported to have died. More than half, of the cases in $2009(n=469,57.3 \%)$ were reported within 20 days of symptom onset. Cases visited 53 countries and were infected in all months of the year, with a peak in September $(n=146)$. By country of residence of the cases, the United Kingdom (UK) reported the highest number of cases $(n=173)$. Italy reported the second highest number of cases $(n=169)$ and was also the country associated with the most cases by country of infection $(n=209)$. A total of 88 new clusters (75 in Europe and 13 outside Europe) were detected in 2009 and were associated with 196 cases. The largest cluster occurred in Italy and involved seven cases. Without the scheme's international database, thirty three $(37.5 \%)$ of the newly detected clusters would not have been identified. In 49 of the accommodation sites with clusters of cases, environmental samples were found to be positive for Legionella spp. Details of 10 sites were published on the European Working Group for Legionella Infections (EWGLI) website for failure to return information on the status of environmental investigations.

\section{Introduction}

The European Surveillance Scheme for Travel Associated Legionnaires' Disease (EWGLINET) was established in 1987 by the European Working Group for Legionella Infections (EWGLI), one year after EWGLI itself was formed. From 1993 to March 2010 the scheme was run as a European Union (EU) funded disease specific network through a coordinating centre in London, United Kingdom (UK), with the common aim among collaborating countries of detection, response, control and prevention of cases and clusters of Legionnaires' disease specifically associated with public accommodation sites used by travellers.
European guidelines for the control and prevention of travel-associated Legionnaires' disease were introduced by EWGLI in 2002, and endorsed by the European Commission in 2003 [1]. They were produced to ensure consistency of approach and a common high standard for investigation of cases and clusters in order to improve protection for travellers throughout Europe.

In April 2010 the scheme was transferred to the European Centre for Disease Prevention and Control (ECDC) and renamed ELDSNet, retaining the original aims and objectives of the network [2]. This paper documents and comments on cases of travel-associated Legionnaires' disease reported to EWGLINET with an onset of illness in 2009.

\section{Methods}

Legionnaires' disease is normally diagnosed in the country of residence of the case and reported from the local or regional level to the country's own national surveillance scheme. Cases that met the microbiological case definitions of the European surveillance scheme [2] were defined as travel-associated if they stayed overnight in a hotel or other type of public accommodation site for at least one night in the two to 10 days before onset of their illness. A secure part of the EWGLI website was used by collaborating countries to electronically report these cases to the international database held by the coordinating centre at the Health Protection Agency (HPA) Centre for Infections in London. Information on the epidemiology, microbiology and travel history of each new case was provided. The database was then searched to determine whether each new case should be classified as a single case or part of a cluster, using the definitions below:

- A single case: a person who stayed at a public accommodation site in the two to 10 days before onset of illness and the site was not associated with any other case of Legionnaires' disease in the previous two years.

- A cluster: two or more cases who stayed at the same public accommodation site in the two to 10 days before onset of illness and whose onsets were within the same two year period. 
These definitions determine the response that is expected by the country where the case became infected within the EU and other EWGLINET (now ELDSNet) countries that have signed up to using EWGLI's guidelines [1]. For single cases, infection could have been acquired from any number of potential sources in the two to 10 days before onset of illness, the accommodation site being just one of them. The guidelines require that the collaborator in the country of infection is informed of the case. He or she must then send the 14 point checklist to the case's accommodation site in order for the site's managers to ensure they are following best practice and one that minimises any potential risk of legionella infection for its guests [1]. This is the only action required at the international level but some countries in the scheme choose to investigate further in accordance with their own national protocols.

Clusters sometimes involve single cases from two or more countries and as such would not normally be recognised as being linked to a specific accommodation site through national surveillance systems alone. It is now the role of the ELDSNet coordinating centre to identify these clusters and ensure they are included in the actions required of all clusters as described below.

When a cluster is detected, all collaborators in the scheme are informed of the incident. A full investigation is required in the country of infection and preliminary results from the risk assessment and start of control measures should be reported back to the coordinating centre within two weeks of the alert, using the guidelines' Form A. A Form B is then used to report the results of environmental sampling and the control measures applied to the site, back to the coordinating centre within a further four weeks, thus allowing six weeks in total for all investigations to be completed. If the forms are not returned within the specified time frames, or they report that actions and control measures are unsatisfactory, ELDSNet publishes the details of the sites associated with the cluster on its website.

\section{FIGURE 1}

Number of travel-associated cases of Legionnaires' disease reported to EWGLINET, 1989-2009 $(\mathrm{n}=8,995)$

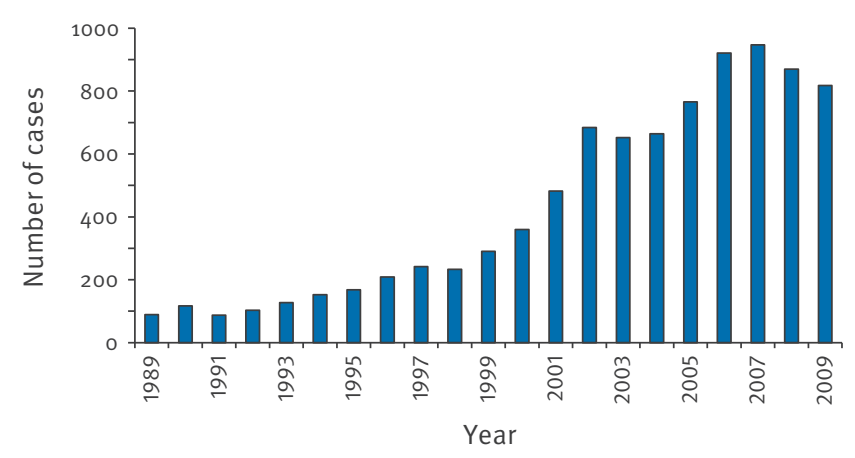

EWGLINET: European Surveillance Scheme for Travel-Associated Legionnaires' Disease
By putting the information in the public domain, individual travellers and tour operators alike can decide for themselves whether or not they wish to contract with these sites. Information is removed from the website when the investigations are reported to have been satisfactorily completed.

Additional cases of Legionnaires' disease are sometimes associated with sites where investigations were reported to have been completed satisfactorily. If these occur within two years of the original cluster, the site becomes a 'reoffender' and a new investigation is required. If a cluster is associated with more than one accommodation site, it is noted as a 'complex cluster' and all sites stayed at by the cluster cases are subject to the investigation procedures as laid down in the guidelines.

Each spring, countries that participate in the scheme are requested to submit their annual dataset of all cases of Legionnaires' disease in residents of their country with onset of illness in the preceding year, together with population data by age group for calculating incidence rates by standardised age groups. Aggregated population data from the countries that reported cases of travel-associated Legionnaires' disease in 2009 was used to calculate incidence rates by standardised age groups for these cases.

\section{Results}

A total of 818 cases of travel associated Legionnaires' disease with onset of infection in 2009 were reported to EWGLINET. This number is 52 cases fewer than the 870 cases reported in 2008 and 129 fewer than when the peak of 947 cases was reported in 2007 (Figure 1). Cases were reported from 22 EWGLINET collaborating countries (United Kingdom (UK) counted as three separate countries) and two countries outside the scheme

TABLE 1

Countries reporting more than 10 cases of travel-associated Legionnaires' disease to EWGLINET in 2008-2009a

\begin{tabular}{|l|c|c|}
\hline \multirow{2}{*}{ Country of report } & \multicolumn{2}{|c|}{ Number of cases } \\
\cline { 2 - 3 } & 2008 & 2009 \\
\hline United Kingdom & 166 & 173 \\
\hline Italy & 127 & 169 \\
\hline France & 191 & 163 \\
\hline The Netherlands & 127 & 109 \\
\hline Spain & 97 & 65 \\
\hline Denmark & 38 & 34 \\
\hline Sweden & 35 & 22 \\
\hline Norway & 21 & 17 \\
\hline Austria & 20 & 17 \\
\hline Belgium & 11 & 12 \\
\hline
\end{tabular}

EWGLINET: European Surveillance Scheme for Travel-Associated Legionnaires' Disease

a A further 14 countries (including the US and New Zealand) reported fewer than 10 cases, and are not listed here. 
(United States (US) and New Zealand). The countries that reported the most cases were the UK $(n=173)$, Italy $(n=169)$, France $(n=163)$, and the Netherlands $(n=109)$ (Table 1). These four countries have consistently reported the highest number of cases to the scheme over several years $[3,4]$.

The mean interval between onset of illness and report to the scheme in 2009 was 29 days (range 2 - 415 days, median 17 days), compared with 27 days in 2008 (range 1-300 days, median 15 days). 176 (21.5\%) cases were reported within 10 days of onset, $469(57.3 \%)$ within 20 days and 606 (74.1\%) within 30 days. The excessive delay in reporting of some cases was due to delays in obtaining and transmitting the required case information from the country's local or regional office to the national collaborating centre and onwards to the EWGLINET scheme.

The male to female ratio in 2009 was 2.7:1 where 597 (73\%) cases were male and $221(27 \%)$ were female. Cases were reported in all age groups (range 19-92 years for males (median 60 years) and 17-88 years (median 64 years) for females). For males the highest number of cases (167) was in the 50-59 year age group whereas for women it was in the 60-69 years age group at 79 cases. Using 2008 population statistics provided by individual EWGLINET countries from their annual return of their national dataset of all cases of Legionnaires' disease, the aggregated age-standardised incidence

\section{FIGURE 2}

Age group and age-standardised incidence rates for cases of travel-associated Legionnaires' disease reported to EWGLINET in 2009 compared with age-standardised incidence rates obtained from the total European dataset of 2008

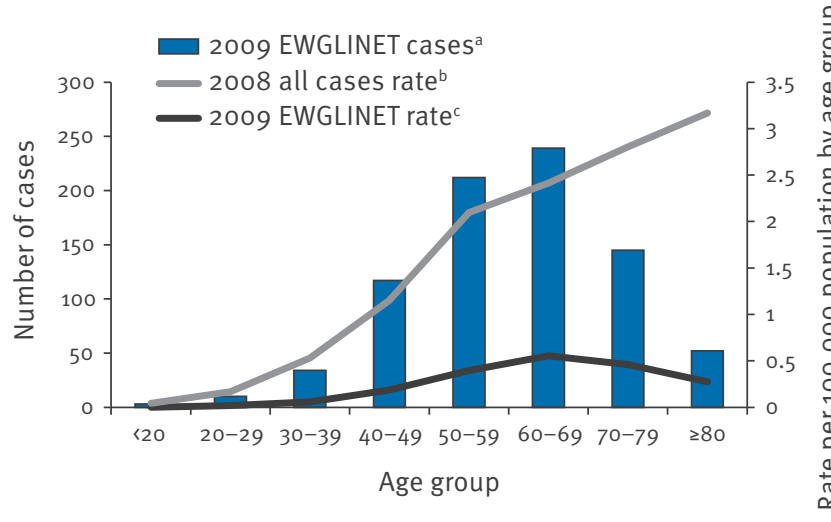

EWGLINET: European Surveillance Scheme for Travel-Associated Legionnaires' Disease

a Number of 2009 EWGLINET cases by age group.

b The 2008 age specific incidence rate is calculated from population data by age group for EWGLINET countries that submitted an annual return of their national dataset to the EWGLINET coordinating centre [6].

The 2009 age specific incidence rates for travel-associated cases was calculated from the 2008 aggregated population data using only those countries that reported cases of travelassociated Legionnaires' disease in 2009. rates for the 22 European countries that reported cases show that rates per 100,000 population by age group increased with increasing age for males and females combined up to age 60-69 years and then decreased again for the 70-79 year-olds and in those aged 80 years or older (Figure 2).

Outcome of illness was reported for $561(68.6 \%)$ cases. Of these cases $28(5 \%)$ were reported to have died, a far lower proportion than the $9.8 \%$ in 2008 . Of those that died in 2009, 20 were males, 17 of whom were between 50 and 79 years of age and eight were females aged between 40 and 89 years. A total of 310 cases $(55.25 \%)$ recovered, and 223 cases (39.75\%) were still ill at time of report. For the remaining 257 cases the outcome was unknown.

The number of cases with Legionnaires' disease normally increases in warmer weather and this travel-associated surveillance scheme highlights this observation. Cases peaked in September $(n=146)$ but occurred in all months of the year (range 20-146 per month).

\section{Microbiological analysis}

On the basis of the EWGLINET case definition, 794 (97.1\%) cases were reported as confirmed cases in 2009. Of these, $82(10 \%)$ were diagnosed by culture of the organism, an increase from $7.7 \%$ in 2008 and $8.2 \%$ in 2007 . Of the culture-confirmed cases, 54 were also diagnosed by urinary antigen detection and a further $701(85.7 \%)$ cases were diagnosed by detection of urinary antigen alone. A total of 11 cases (1.3\%) were confirmed by a four-fold rise in antibody response to L. pneumophila serogroup 1 infection. The remaining 24 $(2.9 \%)$ cases were presumptively diagnosed, 15 (1.8\%) by single high titre and nine (1.1\%) by PCR. Altogether, $712(87 \%)$ cases were reported as L. pneumophila serogroup 1, 13 (1.6\%) as L. pneumophila other serogroup, $73(8.9 \%)$ as L. pneumophila serogroup unknown, one as Legionella other species (L. micdadei or L. bozemanii) and $19(2.3 \%)$ as Legionella species unknown. Of the L. pneumophila other serogroups (sgs), two were $\mathrm{sg}_{2}$, two were sg3, two were $\mathrm{sg}_{5}$, one was $\mathrm{sg} 8$, one

\section{FIGURE 3}

Month of illness onset for cases with Legionnaires' disease reported to EWGLINET, $2009(\mathrm{n}=818)$

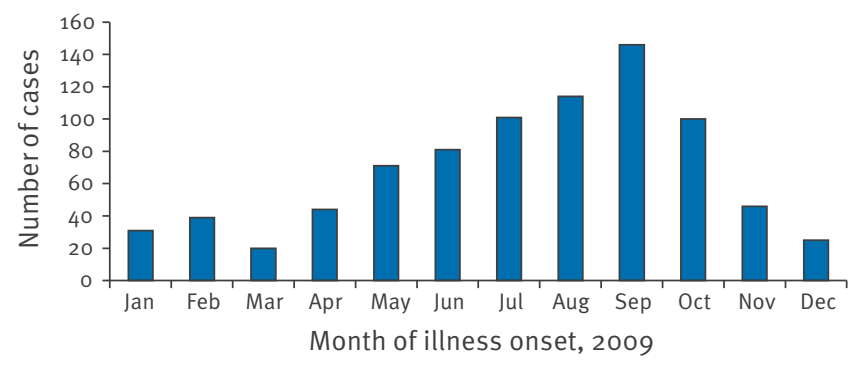

EWGLINET: European Surveillance Scheme for Travel-Associated Legionnaires' Disease 
was sg1-7, one was sg10 and four were sg unknown. PCR was used in conjunction with culture for 22 cases, 18 of which were reported by Denmark, and as a single method of diagnosis for nine cases, seven of which were reported by the Netherlands.

\section{Travel}

Cases visited a total of 53 countries in the 2-10 days before onset of legionella infection. 655 (80\%) cases went to one country only (giving a total of 26 countries visited) in Europe, and $49(6 \%)$ to more than one European country. Ninety six $(11.7 \%)$ cases travelled outside Europe, 88 (10.8\%) to single destinations in 27 countries and eight (1\%) to more than one non-European country. Six cases (0.7\%) went to both European and non-European destinations and 12 (1.5\%) cases were associated with cruise ships. Italy was the country associated with the most cases $(n=209)$ followed by France (135 cases), Spain (92 cases) and Turkey (45 cases).
Different travel patterns emerge when country of report and country of travel are analysed together. The data show that most northern Europeans travel south and become infected abroad whereas many southern European residents have the country of residence and country of infection in common. For instance, France reported that 99 of 135 cases (73\%) who acquired Legionnaires' disease as a result of travel in France were linked to internal travel by French nationals and $\mathbf{2 0} \%$ of the cases that travelled in France were associated with clusters (down from 23.2\% in 2008). Of the 209 cases who acquired Legionnaires' disease in Italy, 125 (60\%) were related to internal travel by Italian nationals and 64 (30.6\%) were associated with clusters (down from 39\% in 2008). Among northern European residents the majority of cases acquired their infection as a result of travel abroad and few cases are associated with their home countries. However, of the 42 cases acquired in the UK, 38 were UK nationals and in the Netherlands 15 of 16 cases acquired in this country were Dutch nationals. The data also show that certain nationals have a preference for travel to particular

\section{FIGURE 4}

Countries visited by cases with travel-associated Legionnaires' disease in 2009, by type of case, EWGLINET data

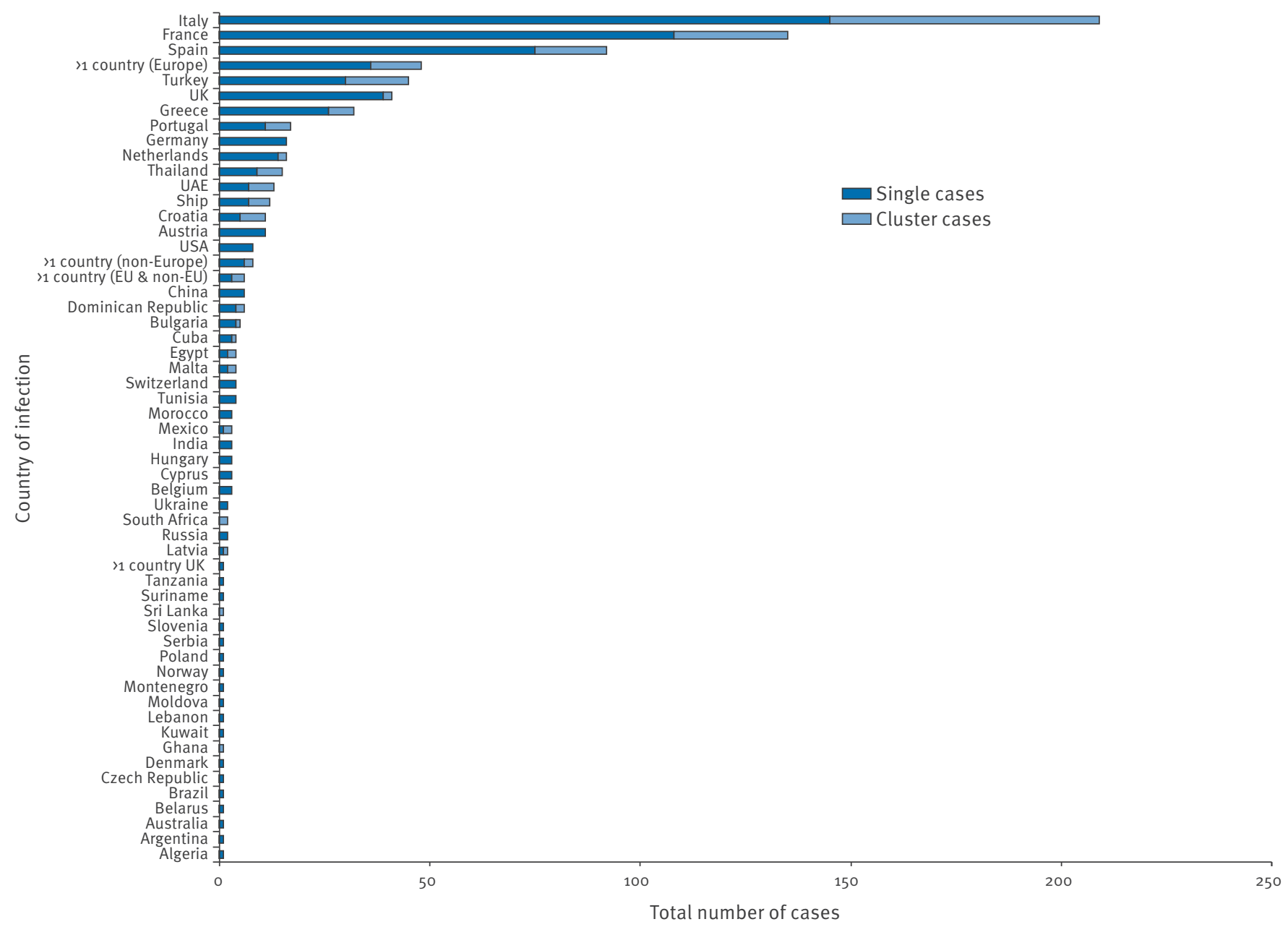


countries. For example 16 (14.7\%) of Dutch cases compared with 16 (9.3\%) of UK cases were linked to travel in Turkey whereas for travel to Spain, the proportion was much higher among UK cases at 31 (18\%) compared with only $6(5.5 \%)$ for Dutch cases.

\section{Clusters}

A total of 88 new clusters (75 in Europe and 13 outside Europe) were detected in 2009 and were associated with 196 (24\%) cases of Legionnaires' disease, five of whom died. Seven cases were associated with the largest cluster in 2009 which occurred in Italy. This country was associated with the highest number of clusters (26) followed by France (16), Turkey (10) and Spain (9). Altogether clusters in Europe occurred in 14 different countries and on two cruise ships. Outside Europe the 13 clusters occurred in nine countries and on one cruise ship. A total of 33 clusters ( $37.5 \%$ ) comprised a single case reported from two or more countries and would not have been detected without the scheme's international database. Clusters were detected in every month of the year but were more common in the months between June and October when 47 (53.4\%) were detected and again in December when nine occurred.

\section{Investigations and publication}

Some of the clusters involved more than one site (complex clusters). In total, 97 sites were linked to the 88 new clusters detected in 2009. Of these sites, 15 were located outside Europe in countries that were not signed up to follow the European guidelines, leaving 82 sites that required EWGLINET investigations. Cluster updates were also issued for 32 'reoffending sites' in 2009 (compared with 35 in 2008). Of the reoffending sites, 20 were situated in Italy, one in France, two in

\section{TABLE 2}

Countries where two or more clusters of travel-associated Legionnaires' disease occurred in 2009, EWGLINET data ${ }^{a}$

\begin{tabular}{|l|c|}
\hline Country of infection & Number of clusters \\
\hline Europe & 26 \\
\hline Italy & 16 \\
\hline France & 10 \\
\hline Turkey & 9 \\
\hline Spain & 3 \\
\hline Portugal & 2 \\
\hline Greece & 2 \\
\hline Not specified & \\
\hline Non-Europe & 2 \\
\hline Cuba & 2 \\
\hline South Africa & 2 \\
\hline Thailand & 2 \\
\hline
\end{tabular}

EWGLINET: European Surveillance Scheme for Travel-Associated Legionnaires' Disease

a A further thirteen countries and one cruise ship were associated with only one cluster and are not listed here.

b Ship
Spain, three in Turkey, two in Greece, and one each in Latvia, Malta, Portugal and Switzerland.

Thus 114 sites in Europe (82 original sites and 32 reoffending sites) required investigation in 2009 according to the procedures outlined in the European guidelines. From these investigations, collaborators reported that Legionella spp. was detected in water samples from 32 of the 82 EWGLINET cluster sites and 17 of the 32 reoffending sites. Thus overall 49 (43\%) sites were positive for Legionella spp., a similar proportion to 2008 when 42.1\% of EWGLINET sites were reported as positive for Legionella spp. No Legionella spp. were detected from $56(49.1 \%)$ investigation sites, six sites were closed and therefore could not be investigated, results were still awaited at two sites and one site had not returned results on the forms $A$ and $B$ within the six-week period specified by the European guidelines.

Although not required to do so, the results of environmental investigations were reported to EWGLINET for eight cluster sites outside Europe. Four of these were in Thailand where three tested positive for L. pneumophila sg1. One site with positive results was reported from a cluster in each of South Africa, the United Arab Emirates and the US. A negative result was reported from a cruise ship cluster investigated in the Middle East.

A total of 10 accommodation sites were published on the EWGLI website in 2009, either due to failure to submit a Form A or B within the specified time period of the European guidelines or because it was reported that the appropriate control measures were not in place. Publishing an accommodation site on the EWGLI website is a means of alerting professionals and the public to the fact that investigation results are unknown or that the control measures have been reported as unsatisfactory. Four of these sites were located in Turkey, three in Italy and one each in Bulgaria, France and Portugal.

\section{Discussion}

Compared with 2008 and 2007, 2009 was associated with a further decrease in travel-associated Legionnaires' disease. This fall may continue to reflect a decrease in the global number of travellers and the impact of the world-wide recession on travel and tourism. More than 922 million travel arrivals worldwide were estimated in 2008 compared with 880 million in 2009 [5]. However, there is also some evidence that improved control and prevention of infection in hotels and other public accommodation sites may be contributing to this decline, particularly where clusters are concerned. The number of detected clusters has fallen from 92 in Europe in 2008 to 75 in 2009 and the overall proportion of cases associated with clusters was at its lowest in 2009 at $24 \%$ compared with $29.1 \%$ in 2008 and $32 \%$ in 2007 . Falls in the number of both single and cluster cases are especially evident in countries that traditionally have a high number of cases such as 
France and Spain. Although Italy was associated with an increase in cases in 2009 compared with 2008 , the proportion of cluster cases there was also down from the year before from $39 \%$ to $31.6 \%$. A far lower proportion of deaths (5\%) were recorded in 2009 from the 561 cases $(68.6 \%)$ with a known outcome compared with the $9.8 \%$ of known deaths in 2008 .

When all cases of Legionnaires' disease are analysed together at the national level, most countries see that the incidence of disease rises by increasing age group when age-standardised rates are calculated [6]. For travel-associated cases age-standardised rates did not show a rise in incidence with increasing age after the age of 69 years, although almost one quarter of the reported cases in both 2008 and 2009 were aged 70 years or more. It is unlikely that under-diagnosis of Legionella spp. infections linked to travel account for the difference in incidence rates for this subset of national cases. Instead it is more likely that relative opportunities for exposure between travel cases and community-acquired cases are different, with only a small proportion of elderly persons travelling from this population age group. However, if the absolute number of travellers among the elderly increases in future years and a higher proportion of cases will occur in this age group, there may well be an associated increase in incidence based on age-standardised rates.

The overall proportion of cluster sites positive for Legionella spp. has remained similar for the last two years at $43 \%$ and $42 \%$ respectively, although positivity rates were higher for the reoffending sites in 2009 compared with 2008. It could be that a plateau has been reached in the level of positive investigation results for new clusters. This may be related to better awareness of control and prevention procedures at these sites and an increased acknowledgement that some clusters occur by chance and that exposure to infection may have occurred elsewhere. With fewer clusters occurring each year, and a smaller proportion of cluster cases in the total dataset, perhaps more attention should now be given to investigating accommodation sites associated with new single cases. These sites would not have been subject to any previous contact with EWGLINET, nor received its advice on minimising risk from Legionella spp. in water systems.

The management of clusters associated with cruise ships is often problematic for EWGLINET as by nature they are more difficult to deal with than clusters in hotels. The ship's sailing itinerary at the time of cluster notification (rather than cluster occurrence) must be established in order to determine through which European country it is appropriate to request investigations. If the ship's itinerary is outside Europe, investigations will be requested through a relevant national public health institute, the World Health Organization (WHO), or via the tour operator or health and safety department of the cruise company. However, opportunities to board the ship and carry out a risk assessment and sampling are usually very limited as the length of time spent in port can be as short as a few hours. EWGLINET has no powers to restrict a ship in port while investigations proceed.

The number of accommodation sites published on the EWGLI website in 2009 fell again compared with 2008 and 2007. Turkey still has a high proportion of its clusters (40\% in $2009,50 \%$ in 2008$)$ appearing on the website for failure to complete investigations on time, but in absolute terms it no longer stands out as a country experiencing problems in meeting the followup requirements as specified in the EWGLI guidelines.

It is encouraging to note that more information on clusters occurring outside Europe has been fed back to EWGLINET. Thailand used the EWGLI guidelines to manage their two clusters in 2009 and completed forms A and $B$, as did health and safety officials involved with the Middle East cruise ship cluster, and the clusters in the United Arab Emirates and South Africa. In the US, the Centers for Disease Control and Prevention also returned information to EWGLINET on their cluster investigations. This feedback has evolved through improved contacts with collaborators in these countries although assistance from WHO is still required to raise awareness of EWGLINET standards elsewhere.

This is the final report of the EWGLINET surveillance scheme in this series; the first report appeared in 1996 [7]. ECDC is now responsible for the scheme, which was renamed ELDSNet in April 2010. The authors hope that ECDC will continue to publish this important data on a regular basis in the future.

\section{Acknowledgements}

This work was funded by the ECDC. We would like to thank all the collaborators for reporting their cases and all persons involved in public health programmes for the control and prevention of travel associated Legionnaires' disease. A list of current collaborating centres can be found on the ECDC website [8].

\section{References}

1. European Working Group for Legionella Infections. European Guidelines for Control and Prevention of Travel Associated Legionnaires' Disease. Available from: http://www.hpa.org.uk/ Topics/InfectiousDiseases/InfectionsAZ/LegionnairesDisease/ Guidlines/legiEuropeanAdviceandGuidance/

2. European Centre for Disease Prevention and Control (ECDC). Stockholm: EWGLINET case definition. Available from: http:// ecdc.europa.eu/en/activities/surveillance/ELDSNet/Pages/ EU\%20case\%2odefinition.aspx

3. Ricketts K, Joseph CA, Yadav R, on behalf of the European Working Group for Legionella Infections. Travel-associated Legionnaires' disease in Europe in 2008. Euro Surveill. 2010;15(21): pii=19578. Available from: http://www. eurosurveillance.org/ViewArticle.aspx?Articleld=19578

4. Joseph CA, Yadav R, Ricketts KD, on behalf of the European Working Group for Legionella Infections. Travel-associated Legionnaires' disease in Europe in 2007. Euro Surveill. 2009;14(18):pii=19196. Available from: http://www. eurosurveillance.org/ViewArticle.aspx?Articleld=19196

5. World Tourism Organization (UNWTO). World Tourism Barometer January 2010. Madrid; 2010. Available from: http://www.unwto.org/facts/eng/pdf/barometer/UNWTO Barom10_1_en_excerpt.pdf. 
6. Joseph CA, Ricketts KD, on behalf of the European Working Group for Legionella Infections. Legionnaires' disease in

Europe 2007-2008. Euro Surveill. 2010;15(8): pii=19493.

Available from: http://www.eurosurveillance.org/ViewArticle. aspx?Articleld $=19493$

7. Hutchinson EJ, Joseph C, Bartlett CL. EWGLI: a European surveillance scheme for travel associated legionnaire's disease. Euro Surveill. 1996;1(5):pii=169. Available from: http://www.eurosurveillance.org/ViewArticle.

aspx?Articleld $=169$

8. European Centre for Disease Prevention and Control (ECDC). [Internet]. Stockholm: ELDSNet. Participating institutions. Available from: http://www.ecdc.europa.eu/en/activities/ surveillance/ELDSNet/Pages/Participating_institutions.aspx 